\title{
Análise bibliométrica da produção científica sobre comunicação alternativa - uma pesquisa na bibliołeca virtual em saúde
}

\author{
Cândice Lima Moreschi* \\ Suzelei Faria Bello** \\ Maria Cristina Piumbato Innocentini Hayashi***
}

\section{Resumo}

Objetivo: identificar, nos trabalhos, aqueles que têm como objetivo de estudo a temática da Comunicação Alternativa, na base de dados da Biblioteca Virtual em Saúde (BVS) de 1981 à 2008. Metodologia: pesquisa documental, qualiquantitativa, nível exploratório-descritivo e envolve as seguintes etapas: 1) seleção do descritor com terminologia definida nos Descritores de Ciência e Saúde (DeCS); 2)levantamento das produções bibliográficas sobre o tema Comunicação Alternativa na BVS; 3) elencados indicadores e suas correlações: ano; autoria e coautoria; país; fonte de publicação; instituições de origem; objetivos e abordagens metodológicas. Resultados: embora as primeiras pesquisas na área sejam da década de 50, apenas nos anos 80 é que começaram a ser, de fato, divulgadas no meio acadêmico. O ano de 1995 merece destaque por ter 6 dos 24 trabalhos analisados desenvolvidos neste ano. $\mathrm{Na}$ análise das autorias dos artigos, $62,5 \%$ foram escritos em autoria múltipla e apenas $37,5 \%$ por um único autor. O país de maior destaque foi os Estados Unidos, onde dezesseis dos vinte e quatro artigos analisados foram escritos. Quanto às fontes de publicação o Journal of Applied Behavior Analysis, contou com 25\% dos trabalhos indexados; quanto as abordagens metodológicas, 50\% dos trabalhos analisados optaram pelo método experimental, 29,16\% pela abordagem documental e 20,83\% baseados na abordagem bibliográfica. Conclusão: Os estudos interdisciplinares, na área da Comunicação Alternativa, merecem destaque; investimento em recursos adequados às necessidades dos usuários de Comunicação Alternativa devem sempre fazer parte do rol de pesquisas.

Palavras-chave: Comunicação Alternativa; Educação Especial; Bibliometria.

\footnotetext{
* Doutorado em andamento em Educação Especial (Educação do Indivíduo Especial). Universidade Federal de São Carlos (UFSCar), São Carlos, São Paulo, Brasil.

** Doutora em Educação Especial, Universidade Federal de São Carlos (UFSCar), São Carlos, São Paulo, Brasil.

*** Professora Doutora na Universidade Federal de São Carlos, Centro de Educação e Ciências Humanas, Departamento de Ciências da Informação. São Carlos, São Paulo, Brasil.
} 


\title{
Bibliometric analysis of scientific production on augmentantive and alternative communication - a research on virtual health library
}

\begin{abstract}
Purpose: To identify the papers whose aim was to study the Alternative Communication issue, of the Virtual Health Library (VHL) database from 1981 to 2008. Methodology: documentary research, qualitative and quantitative exploratory descriptive level, that involves the following steps: 1) selection of the descriptor with terminology defined in the Health Science Descriptors (DeCS); 2) survey of literature production about the topic in the VHL Alternative Communication; 3) list the indicators and their correlation: year, author and co-author, country, source of publication; home institutions; objectives and methodological approaches. Results: Although early research in the area are from the ' 50 s, only in the ' 80 s that they began to be, in fact, disclosed to academy. The year 1995 is noteworthy for having six of the 24 studies analyzed, developed this year. In the analysis of the authorship of articles, $62.5 \%$ were written by multiple authors, and only $37.5 \%$ by a single author. The most prominent country was the United States, where sixteen of the twenty-four articles analyzed were written. Regarding the sources of the publication, Journal of Applied Behavior Analysis had 25\% of jobs indexed; and concerning the methodological approaches, $50 \%$ of the analyzed studies chose the experimental experimental method; $29.16 \%$, documentary approach; and 20.83\% were based on approach literature. Conclusion: The interdisciplinary studies in the area of Alternative Communication are noteworthy; appropriate investment resources to the needs of users of Alternative Communication should always be part of the list of researches.
\end{abstract}

Keywords: Augmentative and Alternative Communication; Special Education; Bibliometric.

\section{Análise bibliométrica: comunicação alternativa}

\section{Introdução}

A educação construiu, ao longo dos anos, uma visão da escola que delimita a escolarização como privilégio de um grupo, sendo a exclusão daqueles que não se enquadravam no padrão homogeneizado proposto algo legitimado nas políticas e práticas educacionais. Nesse contexto, a exclusão tem apresentado características comuns nos processos de segregação e integração que pressupõem a seleção, naturalizando o fracasso escolar (BRASIL, 2008).

A Educação Especial surge no âmbito educacional na LDB 4.024/6l e destaca a necessidade da educação das pessoas com necessidades especiais estarem inseridas na educação geral. A trajetória da Educação Especial foi marcada pelo modelo médico- 
pedagógico, no entanto, a tentativa da Escola Inclusiva, proposta atualmente, é justamente desvincular a imagem da pessoa com necessidades educacionais especiais das concepções do século XIX e início do século XX (JANNUZZI, 2004).

Deste modo, por ser uma ciência multidisciplinar, a Educação Especial, atua com a prevenção, reabilitação e equiparação de oportunidades, considerando as diferentes abordagens (SILVA, 2004). Uma das especialidades que agrega conhecimento para a área é a Fonoaudiologia.

Embora a Fonoaudiologia possua uma herança médico-organicista, no que tange ao perfil de intervenção, apresenta uma base multidisciplinar sobre a qual se edificou, mantendo relações estreitas com outras ciências, como a Linguística e a Educação. Utilizado pelo Fonoaudiólogo, profissional responsável pelo tratamento dos distúrbios da comunicação, os Sistemas de Comunicação Alternativa devem ser considerados como um meio de desenvolver a linguagem e não sendo, em momento algum, responsável por inibir a fala (CHUN; MOREIRA, 1998).

No Brasil, os Sistemas de Comunicação Alternativa (CA) começaram a ser utilizados em instituições de reabilitação para pessoas com paralisia cerebral. No final da década de 50, início da década de 60, surgiu a necessidade de aprimorar a eficiência do atendimento a pacientes que, apesar de frequentarem terapia fonoaudiológica, não desenvolviam suas habilidades comunicativas (GONÇALVES; CAPOVILLA; MACEDO, 1998).

Assim, os Sistemas de Comunicação Alternativa passam a ser utilizados por Fonoaudiólogos como alternativa para desenvolver a linguagem de pessoas desprovidas ou com déficits nas habilidades comunicativas (CHUN; MOREIRA, 1998). Além disso, o Fonoaudiólogo, integrante da equipe multidisciplinar que assiste o usuário de CA, oferece ao paciente a possibilidade de ser incluída em programas existentes em sua comunidade de maneira geral ou em outros, que sejam dedicados à população usuária da CA (BROWNING, 2011).

A expansão do uso dos Sistemas de CA no Brasil ocorreu de maneira gradual e desprovida de uma reflexão sobre o significado da linguagem. A abordagem reducionista, quanto ao tratamento da linguagem, prejudica a terapêutica de pacientes que apresentam alterações nos seus processos comunicativos e limita o uso dos Sistemas de Comunicação (CHUN, 2003).

$\mathrm{Na}$ preocupação em produzir conhecimento científico que discutisse a necessidade da reflexão sobre o significado da linguagem, diversos estudos foram produzidos na área da Comunicação Alternativa, sendo que, nos últimos anos, alguns temas foram amplamente problematizados, tais como: recursos multimídia para avaliação de habilidades cognitivas em pessoas com necessidades especiais (CAPOVILLA; THIERS; CAPOVILLA, 2003); estratégias de ensino naturalístico para implementação e desenvolvimento da linguagem alternativa (NUNES, 2003; PAULA, 1998); análise psicolinguística da comunicação simbólica (NUNES et al., 
2000); percepção das famílias e profissionais sobre o uso de sistemas alternativos de comunicação (NUNES; DELGADO; WALTER, 2011; DELIBERATO, 2011; NUNES; AZEVEDO; FREIRE, 2011).

Outras temáticas, não menos importantes, foram problematizadas por pesquisadores da área:

Bertrán e Basil (2003) realizaram um estudo em que foram utilizados sinais manuais e gráficos, durante a intervenção Fonoaudiológica em grupo, com o intuito de promover a comunicação entre uma criança com Síndrome de Down e deficiência intelectual profunda e seus pares.

Sameshima e Deliberato (2007) buscaramidentificar as habilidades comunicativas utilizadas por um grupo de alunos não-falantes usuários de recursos de CA durante atividade de jogo.

As práticas clínicas, realizadas com adultos usuários de CA apoiadas na CIF (Classificação Internacional de Funcionalidade, Incapacidade e Saúde), foram tema de outra pesquisa na área (PANHAN, 2011). A narrativa desses casos é utilizada na identificação e caracterização de dados relacionados à comunicação e interação social destes indivíduos, com o objetivo de analisar como a CIF pode ser uma ferramenta importante para a pesquisa e clínica na área da CA. Como conclusões, a pesquisadora verificou que o modelo adotado pela CIF é bastante eficiente e permite a promoção de pesquisas em diversas áreas de atuação.

Os estudos, acima citados, demonstram a importância do trabalho em grupo para o desenvolvimento das funções comunicativas de pessoas com déficits na linguagem. A utilização de estratégias de ensino que estejam inseridas no contexto social daquele que passa pelo processo de aprendizagem também é apontado como ponto principal para o êxito de todo o processo. Podemos ainda destacar o sistema de comunicação alternativa - PECS - utilizado em alguns estudos e que trouxe excelentes resultados quanto ao aprendizado de novos recursos de comunicação (WALTER, 2000; 2006; PIZA, 2002; LIMA, 2008). Outro aspecto discutido diz respeito à relação entre o déficit de comunicação dos participantes e os resultados obtidos após a inserção de um novo recurso de comunicação, demonstrando que, mesmo aqueles com importantes alterações na comunicação são capazes de aprender e utilizar um novo recurso de comunicação.

Outro estudo relevante, desenvolvido por Beberiam et al. (2009), objetivou analisar a produção científica vigente a cerca da CA em periódicos de fonoaudiologia, no período de 1997 a 2005, apontou para os poucos estudos na área de Fonoaudiologia e baixa produção científica sobre a temática CA, mas também a necessidade de verificar esse objeto de estudo em outras áreas. 
Nessa perspectiva de compreender como se caracteriza a temática da CA, no contexto de relações com outras áreas, e, em específico, com a Educação Especial, as análises das produções científicas se tornam relevantes, pois permitem elencar temáticas emergentes; observar lacunas e indicar temas para futuras pesquisas.

Vale destacar que os estudos que buscam medir a produção científica podem refletir sobre diferentes áreas e com temas distintos, porém bem definidos e aceitos pela comunidade científica. Uma metodologia para realizar a análise da produção científica é a bibliometria, que pode ser entendida como um instrumento que possibilita minimizar a subjetividade inerente à indexação e recuperação das informações, produzindo conhecimento em determinada área de assunto. Contribui também para tomadas de decisão na gestão da informação e do conhecimento, a medida que pode auxiliar na organização e sistematização dos dados científicos (GUEDES; BORSCHIVER, 2005).

Assim Mugnaini et al. (2006, p. 316), relatam que "para se entender a evolução da ciência, como forma de expressão do conhecimento humano produzido são utilizadas técnicas de medição", que podem ser realizados para estabelecer ou fortalecer indicadores que permitam traçar um perfil do mundo científico.

De acordo com Noronha e Maricato (2008), os estudos bibliométricos estão se tornando cada vez mais frequentes e atraentes, e como mostra Araújo (2006, p. 26), a bibliometria vem se solidificando como uma metodologia que se preocupa com "leituras mais ricas da realidade" que possibilita operacionalizar dados e analisálos, de forma a obter um caminho enriquecedor e confiável.

Todavia Silva, Hayashi, Hayashi (2011) assinalaram que "várias são as áreas de conhecimento que têm lançado mão de métodos e técnicas bibliométricas, resultando em trabalhos que incidem sobre: a literatura de campos científicos ou de assuntos específicos dentro de uma área de conhecimento". Por sua vez, Hayashi, Hayashi, Martinez (2008) comentam que os estudos bibliométricos complementam, de maneira eficaz, as opiniões e os juízos emitidos pelos especialistas de cada área, proporcionando ferramentas úteis e objetivas nos processos de avaliação dos resultados da atividade científica.

Nessa perspectiva, a Bibliometria insere-se como uma ferramenta eficaz que permite obter um espectro abrangente da literatura nacional e internacional voltada aos estudos de Sistemas de Comunicação Alternativa.

\section{Metodologia}

O presente estudo se dispôs a verificar como o tema da CA está representado nos registros de textos completos disponíveis na Biblioteca Virtual em Saúde (BVS) de 1981 a 2008. Para tanto, o desenvolvimento desse estudo priorizou as seguintes etapas: 
1) Revisão da literatura sobre CA e bibliometria para fundamentar teoricamente a pesquisa;

2) Coleta de dados no site da BVS/Bireme; priorizando a utilização do descritor "Auxiliares de Comunicação para Deficientes", sendo selecionados 1648 trabalhos. No entanto, com o objetivo de aprofundar a análise, priorizou-se apenas os textos disponíveis em formato completo, sendo classificados 55 artigos. Desses, sete artigos não estavam disponíveis, e um apresentava duplicidade (uma versão em inglês e outra em português). Assim, foram classificados 47 artigos. Ao iniciarmos a leitura dos textos completos, um novo recorte se fez necessário; foram excluídos da coleta os artigos que versavam sobre aparelhos de amplificação sonora individual, recursos de tecnologia assistiva para adaptação de cadeiras de rodas, estudos sobre exames específicos para caracterizar a atividade cerebral do indivíduo não-falante; perfazendo um total de 24 trabalhos para análise completa.

3) Operacionalização dos dados frente aos seguintes indicadores: ano; idioma; fonte; instituição de origem; autoria e coautoria; objetivos dos estudos; abordagem metodológica utilizada. Os indicadores seguem a seguinte descrição: a). Ano: delinear um panorama de produções/ano; b). Autoria e coautoria: responsável intelectual pela publicação da produção científica e colaborador intelectual, que compartilhou o desenvolvimento da produção científica publicada; c). País:identificar qual o país com maior número de produção sobre a temática; d).Fonte: Direcionar o local onde o trabalho foi encontrado; e). Instituição de origem: verificar a incidência das instituições e seus papéis no desenvolvimento científico da área; f). Objetivo do estudo: propósito que o estudo tende a responder; g). Abordagem metodológica:o caminho metodológico seguido pelos autores

4) Organização e tratamento bibliométrico dos registros coletados por meio da elaboração de tabelas e gráficos e interpretação dos resultados, apoiando-se no referencial teórico sobre produção cientifica de CA para fundamentar a análise obtida.

Vale destacar que a pesquisa proposta envolve a utilização de dados secundários, expressa em artigos científicos o que caracteriza a identificação dos autores como de domínio publico, portanto as questões éticas encontram-se preservadas.

Os dados foram obtidos por meio da pesquisa nas bases de dados indexadas na Biblioteca Virtual em Saúde (BVS), que é composta por diversas bases da América Latina e Caribe.

Para a coleta de dados, como estratégia de busca, utilizamos o sistema DeCS (descritores em ciência e saúde) da BVS que define o descritor "Auxiliares de Comunicação para Deficientes" como sendo "equipamento que propicia às pessoas com deficiência mental ou física um meio de comunicação. O auxilio inclui painéis de demonstração, máquinas de escrever, tubos de raios catódicos, computadores e 
sintetizadores de fala. A saída de tais auxiliares inclui palavras escritas, fala artificial, linguagem de sinais, código Morse e figuras."

Para a coleta de dados, como estratégia de busca, utilizamos o sistema DeCS (descritores em ciência e saúde) da BVS que define o descritor "Auxiliares de Comunicação para Deficientes" como sendo "equipamento que propicia às pessoas com deficiência mental ou física um meio de comunicação. $\mathrm{O}$ auxilio inclui painéis de demonstração, máquinas de escrever, tubos de raios catódicos, computadores e sintetizadores de fala. A saída de tais auxiliares inclui palavras escritas, fala artificial, linguagem de sinais, código Morse e figuras."

\section{Resultados e Discussão}

Os indicadores bibliométricos obtidos permitiram caracterizar e discutir aspectos importantes sobre o tema Comunicação Alternativa. Para melhor visualização desses indicadores foram construídos gráficos e tabelas.

A seguir, na figura l, são apresentados os resultados referentes ao ano de publicação dos 24 artigos completos lidos na íntegra:

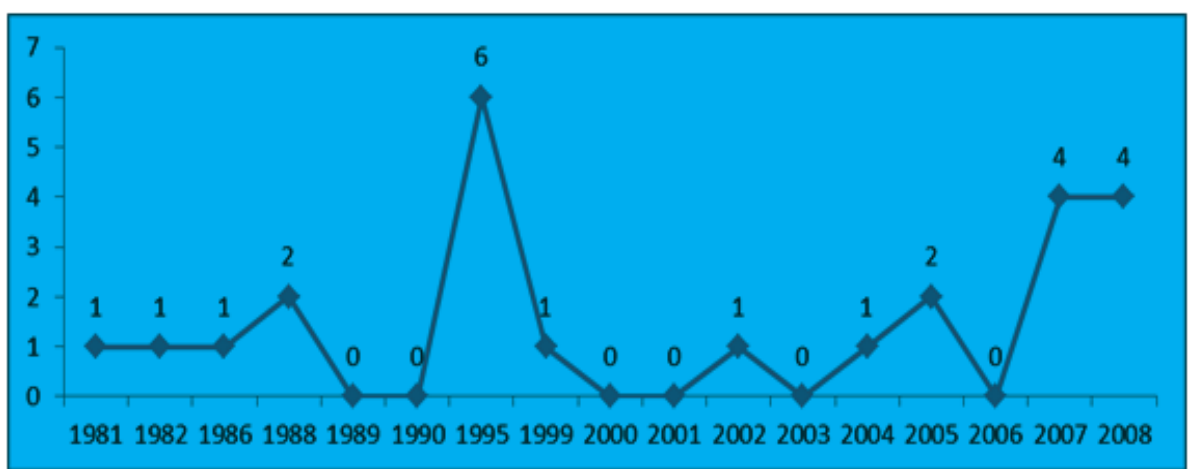

Figura 1. Distribuição dos artigos quanto ao ano.

Na figura 1, identificamos alguns picos de publicação desde a década de 1980. No ano de 1981, as primeiras publicações na área da Comunicação Alternativa foram recuperadas na BVS. Tal resultado indica que, embora as primeiras pesquisas na área sejam da década de 50, apenas nos anos 80, essas pesquisas começaram a ser, de fato, divulgadas no meio acadêmico (GONÇALVES; CAPOVILLA; MACEDO, 1998).

Na década de 1990, o destaque fica para o ano de 1995, quando 6 dos 24 trabalhos foram disponibilizados na BVS. A expansão de trabalhos relacionados à CA, na última década, corrobora com os estudos realizados no âmbito Brasileiro e descritos por CAPOVILLA; THIERS; CAPOVILLA, (2003); NUNES (2003); PAULA (1998); NUNES et al. (2000); SAMESHIMA \& DELIBERATO (2007); LIMA (2009), 
sobre temas que perpassam pela avaliação cognitiva de crianças, caracterização e uso dos sistemas de CA em diferentes contextos, desenvolvimento de estratégias de uso dos recursos de CA por meio do ensino naturalísticos.

A tendência para os próximos anos é a crescente demanda nas publicações na área de Comunicação Alternativa, uma vez que diversos grupos de pesquisas vêm se consolidando no sentido de desenvolver pesquisas na área. Atualmente, projetos de pesquisa vêm sendo desenvolvidos no Laboratório de Tecnologia e Comunicação Alternativa (LATECA), na Universidade do Estado do Rio de Janeiro, nas áreas de desenvolvimento humano, linguagem e comunicação, interação social, comunicação alternativa e educação de pessoas com deficiência. Na UNESP-Marília, há um grupo de pesquisadores realizando estudos que versam sobre comunicação alternativa, educação especial, formação de profissionais da saúde e da educação no processo inclusivo do deficiente. Na Universidade Federal do Rio de Janeiro, outro grupo de pesquisa desenvolve projetos na área de tecnologia assistiva voltada aos recursos de CA no ambiente escolar e hospitalar, além de trabalhar com formação de professores. Na Universidade Federal de São Carlos, são conduzidas investigações na área de deficiência intelectual, autismo, inclusão, profissionalização e comunicação alternativa. Outros centros acadêmicos também possuem pesquisadores envolvidos com a temática da CA, tais como a Universidade Federal do Rio Grande do Norte e a Universidade de Campinas - Unicamp. Essas pesquisas são conduzidas por pesquisadores que possuem importante produção científica no campo da Educação Especial, com destaque para trabalhos relacionados a CA (NUNES; PELOSI; WALTER, 201l; DELIBERATO; GONCALVES; MACEDO, 2009; PELOSI, 2003; WALTER; ALMEIDA, 2010; REILY, 2009).

No presente estudo, foi possível verificar a distribuição dos trabalhos realizados em autoria individual e co-autoria, conforme apontam os dados da Figura 2.

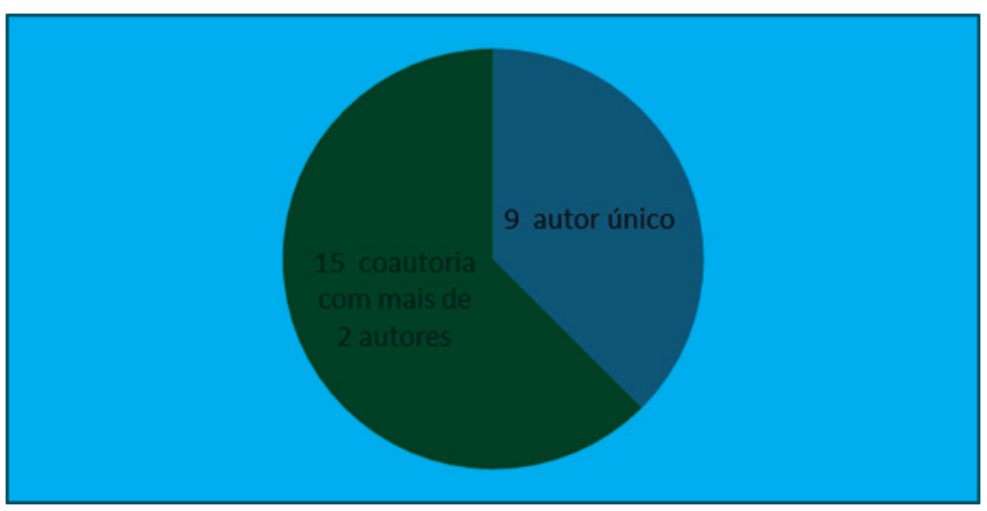

Figura 2. Trabalhos realizados em autoria individual 
Na figura 2, pode-se observar que $62,5 \%$ dos artigos são em autoria múltipla e apenas $37,5 \%$ foi realizado por um único autor. A co-autoria indica uma tendência em se estabelecer parcerias entre os pesquisadores com o intuito de desenvolver pesquisas na temática estudada.

A figura a seguir apresenta os resultados referentes ao País de origem das publicações analisadas.

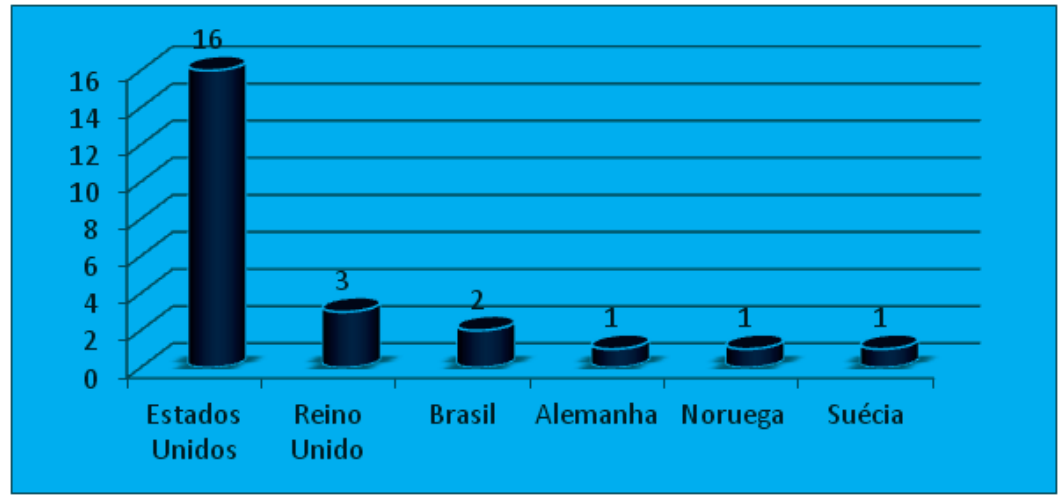

Figura 3. País de origem dos artigos completos

Ao observarmos a Figura 3, verificamos que a maioria dos artigos sobre a temática da CA foram publicados nos Estados Unidos, com destaque para o Journal of Applied Behavior Analysis, com 25\% dos trabalhos publicados e outros periódicos como demonstra a Figura 4.

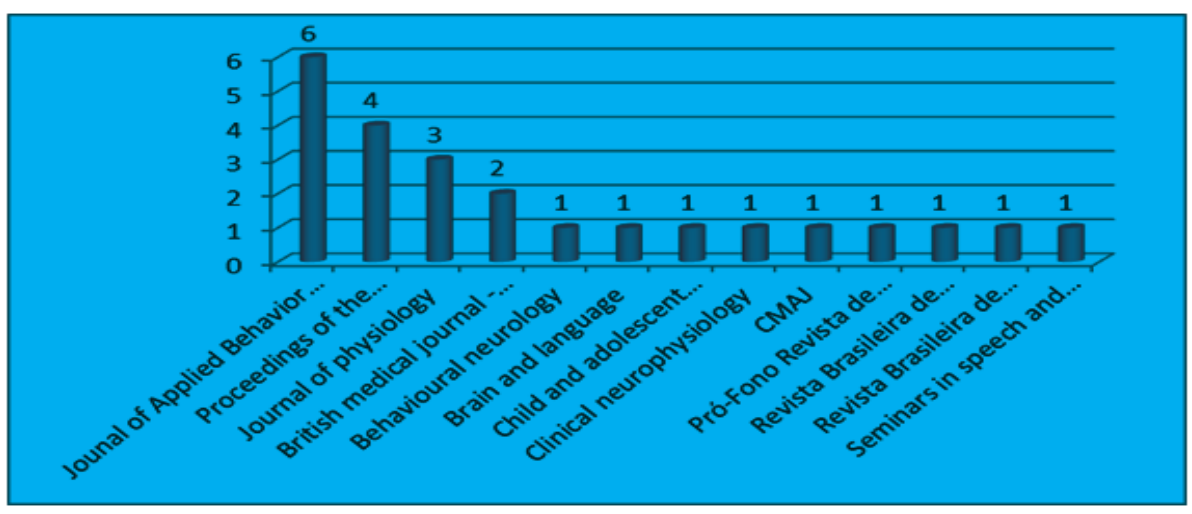

Figura 4. Fonte de publicação dos artigos completos 
Com relação às instituições as quais os autores dos trabalhos estão vinculados observamos no Quadro 2que elas totalizam 24 instituições diferentes o que remete ao fato de que a temática da CA tem sido discutida em diversos centros universitários. Tal fato contribui de maneira positiva para que novas tecnologias sejam desenvolvidas em prol daqueles que utilizam os recursos alternativos de comunicação para interagir no meio em que vivem.

Quadro l - Distribuição dos trabalhos, de acordo com a instituição de origem, autores e ano

\begin{tabular}{|c|c|}
\hline Instituição/departamento & Autores \\
\hline $\begin{array}{l}\text { Clinical and Health Psychology Research } \\
\text { Centre, School of Human and Life Sciences, } \\
\text { Roehampton University }\end{array}$ & Kubler, A.; Birbaumer, N.(2008) \\
\hline Yale ChildStudy Center & Paul, R. (2008) \\
\hline University of Maryland School of Medicine & $\begin{array}{l}\text { Linebarger, M.; Mccall, D.; Virata, T.; } \\
\text { Berndt, R. S. (2007) }\end{array}$ \\
\hline $\begin{array}{l}\text { Pós-Graduação em Educação Especial da } \\
\text { Universidade Federal de São Carlos }\end{array}$ & $\begin{array}{l}\text { Almeida, M.A.; Piza M.H.M.; Lamônica } \\
\text { D.A.C. (2005) }\end{array}$ \\
\hline $\begin{array}{l}\text { Department of Neurology, Boston University } \\
\text { School of Medicine and the Veterans Affairs } \\
\text { Boston Healthcare System }\end{array}$ & $\begin{array}{l}\text { Kurland, J.; Naeser, M.A.; Baker, E.H.; } \\
\text { Doron, K.; Martin, P.I.; Seekins, H.E.; } \\
\text { Bogdan, A.; Renshaw, P.; Yurgelun-Todd, D. } \\
\text { (2004) }\end{array}$ \\
\hline ClaremontMckennaCollege & $\begin{array}{l}\text { Charlop-Christy, M.H.; Carpenter, M.; Le } \\
\text { L.; Leblanc, L.A.; Kellet, K. (2002) }\end{array}$ \\
\hline University at Albany & Durand, V.M. (1999) \\
\hline $\begin{array}{l}\text { Department of Communicative Disorders, } \\
\text { University of Wisconsin }\end{array}$ & $\begin{array}{l}\text { Hustad, K.C.; Keppner, K; Schanz, A.; Berg, } \\
\text { A. (2008) }\end{array}$ \\
\hline $\begin{array}{l}\text { Institute of Medical Psychology and } \\
\text { Behavioural Neurobiology, University of } \\
\text { Tuebingen }\end{array}$ & Birbaumer, N.; Cohen, L.G. (2007) \\
\hline $\begin{array}{l}\text { Department of Neuroscience, Division of } \\
\text { Engineering, Department of Computer Science, } \\
\text { Brown University, Providence }\end{array}$ & $\begin{array}{l}\text { Donoghue ,J.P.; Nurmikko, A.; Black, M.; } \\
\text { Hochberg, L.R. (2007) }\end{array}$ \\
\hline $\begin{array}{l}\text { Wadsworth Center, Laboratory of Nervous } \\
\text { System Disorders, New York State Department } \\
\text { of Health and State University of New York, } \\
\text { Albany }\end{array}$ & Wolpaw, J.R. (2007) \\
\hline George Mason Universy & Schepis, M.M.; Reid, D.H. (1995) \\
\hline $\begin{array}{l}\text { Division of Rehabilitation Sciences, University } \\
\text { of Oklahoma Health Sciences Center }\end{array}$ & $\begin{array}{l}\text { Schlosser, R.W.; Belfiore P.J.; Nigam, R.; } \\
\text { Blischak, D.; Hetzroni, O. (1995) }\end{array}$ \\
\hline Department of Psychology, University of Oslo & $\begin{array}{l}\text { Tetzchner, S.V.; Brekke, K.M.; Sjøthun, B.; } \\
\text { Grindheim; E.(2005) }\end{array}$ \\
\hline
\end{tabular}


continuação quadro 1

\begin{tabular}{|l|l|}
\hline $\begin{array}{l}\text { Pontifícia Universidade Católica de São Paulo } \\
\text { - PUC-SP }\end{array}$ & Fidalgo, A.P.; Godoi, J.P; Gioia, O.S. (2008) \\
\hline $\begin{array}{l}\text { The University of Iowa and Des Moines Public } \\
\text { Schools }\end{array}$ & $\begin{array}{l}\text { Wacker, D.P; Wiggins, B. Fowler, M.; Berg, } \\
\text { W.K. (1988) }\end{array}$ \\
\hline $\begin{array}{l}\text { United Cerebral Palsy, INC., The John } \\
\text { F. Kennedy Institute and Johns Hopkins } \\
\text { School of Medicine, and Western Michigan } \\
\text { University }\end{array}$ & Hurlbut, B.I.; Iwata, B.A.; Green, J.D. (1992) \\
\hline $\begin{array}{l}\text { Department of Computer and Information } \\
\text { Science, University of Pennsylvania, } \\
\text { Philadelphia }\end{array}$ & Liberman, M. (1995) \\
\hline $\begin{array}{l}\text { Department of Speech Communication } \\
\text { and Music Acoustics, Royal Institute of } \\
\text { Technology, Stockholm, Sweden }\end{array}$ & Carlson, R. (1995) \\
\hline $\begin{array}{l}\text { Research Laboratory of Electronics, } \\
\text { Massachusetts Institute of Technology, } \\
\text { Cambridge }\end{array}$ & Allen, J. (1995) \\
\hline Seelbach Associates & Seelbach, C. (1995) \\
\hline Departmentof Speech Therapy & Perry, A.R.; Gawel, M.; Rose, F.C.(1981) \\
\hline $\begin{array}{l}\text { Speech Therapy Department, Frenchay } \\
\text { Hospital, Bristol }\end{array}$ & Easton, J. (1988) \\
\hline & Lee, R. E. (1986) \\
\hline
\end{tabular}

Os indicadores construídos permitiram identificar os objetivos e as abordagens metodológicas presentes nos 24 artigos analisados, conforme mostram os resultados descritos no Quadro 3 destacamos os objetivos e as abordagens metodológicas utilizadas nos trabalhos.

Quadro 2 - Objetivos e abordagens metodológicas dos artigos

\begin{tabular}{|l|l|l|l|}
\hline \multicolumn{1}{|c|}{ Autores } & \multicolumn{1}{|c|}{ Objetivo do Trabalho } & \multicolumn{2}{c|}{ Abordagem Metodológica } \\
\hline Kubler A & $\begin{array}{l}\text { Investigar a relação entre } \\
\text { comprometimento físico e o } \\
\text { desempenho de um sistema } \\
\text { cérebro-computador (BCI) }\end{array}$ & $\begin{array}{l}\text { Pesquisa } \\
\text { experimental }\end{array}$ & $\begin{array}{l}\text { Observação; Utilização de } \\
\text { testes: sistema cérebro- } \\
\text { computador (BCI-training) }\end{array}$ \\
\hline $\begin{array}{l}\text { Linebarger } \\
\text { McCall D }\end{array}$ & $\begin{array}{l}\text { Investigar o impacto do uso } \\
\text { do sistema SentenceShaper } \\
\text { na narrativa de seis afásicos } \\
\text { não-fluentes. }\end{array}$ & $\begin{array}{l}\text { Pesquisa } \\
\text { Experimental }\end{array}$ & $\begin{array}{l}\text { Observação; Utilização de } \\
\text { testes: Análise Quantitativa } \\
\text { de Produção (QPA; Saffranet } \\
\text { al., 1989); Análise da Unidade } \\
\text { de Informação Correta (CIU; } \\
\text { Virata T }\end{array}$ \\
Berndt RS
\end{tabular}


continuação quadro 2

\begin{tabular}{|c|c|c|c|}
\hline $\begin{array}{l}\text { Almeida MA } \\
\text { Piza MHM } \\
\text { Lamônica } \\
\text { DAC }\end{array}$ & $\begin{array}{l}\text { Avaliar a eficácia do (Pecs- } \\
\text { Adaptado) e do Picture } \\
\text { Communication Symbols } \\
\text { (PCS) na comunicação de } \\
\text { paralítico cerebral. }\end{array}$ & $\begin{array}{l}\text { Estudo } \\
\text { Experimental } \\
\text { AB (sujeito } \\
\text { como próprio } \\
\text { Controle) }\end{array}$ & Programa de Intervenção \\
\hline $\begin{array}{l}\text { Kurland J } \\
\text { Naeser MA } \\
\text { Baker EH } \\
\text { Doron K } \\
\text { Martin PI } \\
\text { Seekins HE } \\
\text { Bogdan A } \\
\text { Renshaw P } \\
\text { Yurgelun- } \\
\text { Todd D }\end{array}$ & $\begin{array}{l}\text { Analisar os dados de uma } \\
\text { Ressonância Magnética } \\
\text { Funcional (fMRI), obtidos a } \\
\text { partir da execução de tarefas } \\
\text { semânticas de um indivíduo } \\
\text { afásico em situação } \\
\text { individual e interativa com } \\
\text { outro afásico }\end{array}$ & $\begin{array}{l}\text { Estudo } \\
\text { Experimental }\end{array}$ & $\begin{array}{l}\text { Observação; Aplicação } \\
\text { de testes: Ressonância } \\
\text { Magnética Funcional (fMRI). }\end{array}$ \\
\hline $\begin{array}{l}\text { Charlop- } \\
\text { Christy MH } \\
\text { Carpenter M } \\
\text { Le L } \\
\text { LeBlanc LA } \\
\text { Kellet K }\end{array}$ & $\begin{array}{l}\text { Avaliar a aquisição e } \\
\text { utilização do PECS por três } \\
\text { crianças autistas. }\end{array}$ & $\begin{array}{l}\text { Estudo } \\
\text { Experimental; } \\
\text { Linha de Base } \\
\text { Múltipla }\end{array}$ & $\begin{array}{l}\text { Observação; Aplicação de } \\
\text { Programa de Intervenção: } \\
\text { Picture Exchange } \\
\text { Communication system } \\
\text { (PECS; Bondy\&EFrost, 1994). }\end{array}$ \\
\hline Durand VM & $\begin{array}{l}\text { Avaliar a efetividade } \\
\text { do treinamento de } \\
\text { comunicação funcional } \\
\text { (FCT) na intervenção com } \\
\text { alunos com deficiências } \\
\text { severas. }\end{array}$ & $\begin{array}{l}\text { Estudo } \\
\text { Experimental; } \\
\text { Linha de Base } \\
\text { Múltipla } \\
\text { cruzando entre } \\
\text { sujeitos } \\
\end{array}$ & $\begin{array}{l}\text { Observação; Aplicação de } \\
\text { Programa de Intervenção: } \\
\text { Treinamento de Comunicação } \\
\text { Funcional (FCT; } \\
\text { Carr\&DDurand, 1985). }\end{array}$ \\
\hline $\begin{array}{l}\text { Schepis MM } \\
\text { Reid DH }\end{array}$ & $\begin{array}{l}\text { Examinar os efeitos da } \\
\text { utilização do VOCA (voice } \\
\text { output communication } \\
\text { aid) por um indivíduo com } \\
\text { deficiência grave durante } \\
\text { interações comunicativas } \\
\text { com pessoa de apoio } \\
\text { (interlocutor) }\end{array}$ & $\begin{array}{l}\text { Pesquisa } \\
\text { Experimental; } \\
\text { múltiplas } \\
\text { sondagens }\end{array}$ & $\begin{array}{l}\text { Observação; Aplicação de } \\
\text { Programa de Intervenção: } \\
\text { VOCA }\end{array}$ \\
\hline
\end{tabular}


continuação quadro 2

\begin{tabular}{|c|c|c|c|}
\hline $\begin{array}{l}\text { Schlosser } \\
\text { RW } \\
\text { Belfiore PJ } \\
\text { Nigam R } \\
\text { Blischak D } \\
\text { Hetzroni O }\end{array}$ & $\begin{array}{l}\text { Analisar os efeitos da } \\
\text { inserção de estímulos } \\
\text { auditivos em forma de } \\
\text { discurso sintético na } \\
\text { aprendizagem de símbolos } \\
\text { gráficos. }\end{array}$ & $\begin{array}{l}\text { Estudo } \\
\text { Experimental; } \\
\text { delineamento } \\
\text { de tratamentos } \\
\text { paralelos }\end{array}$ & $\begin{array}{l}\text { Observação; Aplicação de } \\
\text { Programa de Intervenção }\end{array}$ \\
\hline $\begin{array}{l}\text { Fidalgo AP } \\
\text { Godoi JP } \\
\text { Gioia OS }\end{array}$ & $\begin{array}{l}\text { Aplicar e avaliar a } \\
\text { eficácia do sistema } \\
\text { PECS na intervenção em } \\
\text { comunicação com criança } \\
\text { com Síndrome do X-Frágil } \\
\text { e adulto com Síndrome de } \\
\text { Down. }\end{array}$ & $\begin{array}{l}\text { Pesquisa } \\
\text { Experimental }\end{array}$ & $\begin{array}{l}\text { Observação; Aplicação de } \\
\text { programa de intervenção: } \\
\text { PECS (Bondy\&\&Frost, 1994). }\end{array}$ \\
\hline $\begin{array}{l}\text { Wacker DP } \\
\text { Wiggins B } \\
\text { Fowler M } \\
\text { Berg WK }\end{array}$ & $\begin{array}{l}\text { Avaliar o uso de } \\
\text { microswitches por alunos } \\
\text { deficientes múltiplos na } \\
\text { demonstração de desejos e } \\
\text { sentimentos. }\end{array}$ & $\begin{array}{l}\text { Pesquisa } \\
\text { Experimental }\end{array}$ & $\begin{array}{l}\text { Observação; Aplicação de } \\
\text { procedimento de intervenção }\end{array}$ \\
\hline $\begin{array}{l}\text { Hurlbut BI } \\
\text { Iwata BA } \\
\text { Green JD }\end{array}$ & $\begin{array}{l}\text { Comparar o treinamento de } \\
\text { dois sistemas de linguagem } \\
\text { (sistema BLISS e sistema de } \\
\text { figuras icônicas) destinado } \\
\text { a três adolescentes com } \\
\text { deficiência grave. }\end{array}$ & $\begin{array}{l}\text { Pesquisa } \\
\text { experimental }\end{array}$ & $\begin{array}{l}\text { Observação; Aplicação de } \\
\text { procedimento de intervenção }\end{array}$ \\
\hline $\begin{array}{l}\text { Perry AR } \\
\text { Gawel M } \\
\text { Rose FC }\end{array}$ & $\begin{array}{l}\text { Descrever a avaliação de } \\
\text { dispositivos de comunicação } \\
\text { existentes no mercado por } \\
\text { pacientes com disfunção } \\
\text { lateral amiotrófica. }\end{array}$ & $\begin{array}{l}\text { Pesquisa } \\
\text { Experimental }\end{array}$ & $\begin{array}{l}\text { Observação; Utilização de } \\
\text { testes: ffFrenchaydysarthria } \\
\text { assessment (Enderby,1979); } \\
\text { dispositivos de comunicação } \\
\left(\text { Splink }{ }^{\circledR}, \text { Canon }\right. \\
\text { Comunicator }^{\circledR}, \text { Lightwriter }{ }^{\circledR} \text {, } \\
\text { Edu-Comm }^{\circledR} \text { ) }\end{array}$ \\
\hline
\end{tabular}


continuação quadro 2

\begin{tabular}{|c|c|c|c|}
\hline Paul R & $\begin{array}{l}\text { Revisão de programas } \\
\text { sobre o desenvolvimento } \\
\text { da linguagem de crianças } \\
\text { com autismo de alto } \\
\text { funcionamento }\end{array}$ & $\begin{array}{l}\text { Pesquisa } \\
\text { bibliográfica }\end{array}$ & Bibliográfica \\
\hline $\begin{array}{l}\text { Birbaumer N } \\
\text { Cohen LG }\end{array}$ & $\begin{array}{l}\text { Discutir o estado da arte de } \\
\text { pesquisas sobre a interface } \\
\text { cérebro-computador em } \\
\text { pacientes com severo } \\
\text { comprometimento físico }\end{array}$ & $\begin{array}{l}\text { Pesquisa } \\
\text { Bibliográfica }\end{array}$ & Bibliográfica \\
\hline $\begin{array}{l}\text { Donoghue JP } \\
\text { Nurmikko A } \\
\text { Black M } \\
\text { Hochberg } \\
\text { LR }\end{array}$ & $\begin{array}{l}\text { Descrever a lógica, os } \\
\text { estágios iniciais de } \\
\text { desenvolvimento, e as } \\
\text { aplicações iniciais em seres } \\
\text { humanos de sistemas de } \\
\text { interface neural (NISs) para } \\
\text { seres humanos com paralisia }\end{array}$ & $\begin{array}{l}\text { Pesquisa } \\
\text { Bibliográfica }\end{array}$ & Bibliográfica \\
\hline Wolpaw JR & $\begin{array}{l}\text { Discutir a importância } \\
\text { dos sistemas cérebro- } \\
\text { computador (BCI) como } \\
\text { procedimentos para a } \\
\text { comunicação e controle } \\
\text { de pessoas com severos } \\
\text { comprometimentos motores. }\end{array}$ & $\begin{array}{l}\text { Pesquisa } \\
\text { Bibliográfica }\end{array}$ & Bibliográfica \\
\hline $\begin{array}{l}\text { Tetzchner } \\
\text { SV } \\
\text { Brekke KM } \\
\text { SjØthun B } \\
\text { Grindheim E }\end{array}$ & $\begin{array}{l}\text { Discutir sobre o } \\
\text { desenvolvimento de } \\
\text { linguagem alternativa em } \\
\text { crianças à partir de práticas } \\
\text { inclusivas. }\end{array}$ & $\begin{array}{l}\text { Pesquisa } \\
\text { Bibliográfica }\end{array}$ & Bibliográfica \\
\hline $\begin{array}{l}\text { Hustad KC } \\
\text { Keppner K } \\
\text { Schanz A } \\
\text { Berg A }\end{array}$ & $\begin{array}{l}\text { Descrever pesquisas } \\
\text { sobre intervenções } \\
\text { fonoaudiológicas em pré- } \\
\text { escolares que necessitam de } \\
\text { recursos de comunicação } \\
\text { alternativa. }\end{array}$ & $\begin{array}{l}\text { Pesquisa } \\
\text { Documental }\end{array}$ & Documentação Indireta \\
\hline
\end{tabular}


continuação quadro 2

\begin{tabular}{|c|c|c|c|}
\hline Liberman M & $\begin{array}{l}\text { Discutir sobre a situação } \\
\text { atual e perspectivas dos } \\
\text { computadores de fala } \\
\text { sintetizada }\end{array}$ & $\begin{array}{l}\text { Pesquisa } \\
\text { documental }\end{array}$ & Documentação Indireta \\
\hline Carlson R & $\begin{array}{l}\text { Discutir sobre metodologias } \\
\text { utilizadas na produção de } \\
\text { discurso sintético por meio } \\
\text { de um sistema de "texto } \\
\text { para fala". }\end{array}$ & $\begin{array}{l}\text { Pesquisa } \\
\text { documental }\end{array}$ & Documentação Indireta \\
\hline Allen J & $\begin{array}{l}\text { Discutir sobre os } \\
\text { aspectos linguísticos dos } \\
\text { sintetizadores de fala }\end{array}$ & $\begin{array}{l}\text { Pesquisa } \\
\text { documental }\end{array}$ & Documentação Indireta \\
\hline Seelbach C & $\begin{array}{l}\text { Discutir os esforços para } \\
\text { comercializar tecnologias } \\
\text { de fala em dois mercados: } \\
\text { telecomunicações e auxílios } \\
\text { aos deficientes }\end{array}$ & $\begin{array}{l}\text { Pesquisa } \\
\text { documental }\end{array}$ & Documentação Indireta \\
\hline Easton J & $\begin{array}{l}\text { Apresentar os principais } \\
\text { aspectos sobre comunicação } \\
\text { alternativa (população } \\
\text { beneficiada e recursos) }\end{array}$ & $\begin{array}{l}\text { Pesquisa } \\
\text { documental }\end{array}$ & Documentação Indireta \\
\hline Lee RE & $\begin{array}{l}\text { Discutir os possíveis } \\
\text { benefícios do uso do } \\
\text { microcomputador as } \\
\text { pessoas com déficits na } \\
\text { comunicação oral. }\end{array}$ & $\begin{array}{l}\text { Pesquisa } \\
\text { documental }\end{array}$ & Documentação Indireta \\
\hline
\end{tabular}

O Quadro 3 mostra que há presença majoritária do delineamento experimental com 12 artigos (50\% do total). Em 7/24 artigos (29\%), a pesquisa documental esteve presente e a pesquisa bibliográfica foi adotada em 5 artigos (21\%) analisados. A prevalência da pesquisa experimental pode estar relacionada com o intuito de desenvolver melhores estratégias de ensino-aprendizagem das técnicas voltadas à CA. Pesquisas que envolvam análise documental ou bibliográfica têm sido desenvolvidas com a finalidade de mapear os percursos percorridos pelos pesquisadores da área.

\section{Conclusões}

O estudo bibliométrico realizado, aponta para a prevalência entre os artigos de estudos internacionais (principalmente norte-americanos) em relação aos realizados no Brasil. Isso reflete o amadurecimento das pesquisas norte-americanas em relação a temática de CA e mostra que ainda há um longo caminho a percorrer nesse campo no Brasil. 
Outra conclusão do estudo é que embora existam registros de artigos desde a década de 1980, foi na década de 2000 que houve um aumento significativo da produção científica na área em todo o mundo.

Verificou-se, ainda, que as pesquisas desenvolvidas adotam tanto o delineamento experimental quanto documental e bibliográfico, sendo que todos contribuem para traçar um panorama prático e teórico da temática da comunicação alternativa.

Esperamos que os indicadores bibliométricos, produzidos neste estudo, tenham contribuído para traçar um perfil da área da Comunicação Alternativa presente na BVS, por meio da identificação das temáticas mais estudadas e tipos de estudos realizados. Os dados provenientes deste estudo sugerem a necessidade de realização de mais estudos sobre o tema para que contribuam com o avanço do conhecimento na área. Uma ausência notada, nessa análise, foi em relação aos estudos interdisciplinares, que devem merecer destaque, e sobre o investimento em recursos adequados às necessidades dos usuários de CA. Tais lacunas podem se constituir em temas de pesquisas, visando incrementar a produção científica nessa importante área.

\section{Referências}

ARAÚJO, C. A. Bibliometria: evolução histórica e questões atuais. Em Questão. 2006;12(1):1132.

BERBERIAN, A. P.; KRÜGER, S.; GUARINELLO, A. C.; MASSI, G. A. A Produção do conhecimento em Fonoaudiologia em Comunicação Suplementar e/ou Alternativa: análise de periódicos. Rev. CEFAC. 2009;11(2):258-266.

BERTRÁN, A.; BASIL, C. Rubén: uso de sinais manuais e gráficos no processo de escolarização de uma criança com Síndrome de Down. In: ALMIRALL, C.B.; SORO-CAMATS, E.; BULTÓ, C.R. Sistemas de sinais e ajudas técnicas para a comunicação alternativa e a escrita princípios teóricos e aplicações. São Paulo: Santos; 2003, p. 149-158.

BRASIL. Ministério da Educação. Secretaria de Educação Especial. Política Nacional de Educação Especial na Perspectiva da Educação Inclusiva. Brasília: MEC/SEESP. 2008.

BROWNING, N. Compartilhando experiências. In: NUNES, L. R. O. P.; PELOSI, M. B.; WALTER, C. C. F. Compartilhando experiências: ampliando a comunicação alternativa. Marília: ABPEE; 2011, p. 7-16.

CAPOVILLA, F. C.; THIERS, V.O.; CAPOVILLA, A.G.S. Avaliação cognitiva de crianças com severos distúrbios motores: versões computadorizadas, normatizadas e validadas de cinco testes de vocabulário, compreensão auditiva, prontidão para leitura e inteligência geral. In: NUNES, L.R.O.P. (Org.). Favorecendo o desenvolvimento da comunicação em crianças e jovens com necessidades educacionais especiais. Rio de Janeiro: Dunya; 2003, p. 253-262.

CHUN, R. Y. S. Comunicação suplementar e/ou alternativa: favorecimento da linguagem de um participante não falante. Pró-fono R. Atual. Cient. 2003;15(1):55-64. 
CHUN, R. Y. S. Moreira E. C. Questões da linguagem e da tecnologia na comunicação suplementar e/ou alternativa. In: FOZ, F. B; PICCARONE, M. L. C. D.; BURSZTYN, C.S. (Org.). A tecnologia informática na fonoaudiologia. São Paulo: Plexus; 1998, p. 97-113.

DELIBERATO, D. Percepção de mães e fonoaudiólogos a respeito do uso de sistemas suplementares e alternativos de comunicação. In: NUNES, L.R.O.P.; PELOSI, M.B.; WALTER, C. C. F. Compartilhando experiências: ampliando a comunicação alternativa. Marília: ABPEE; 2011, p. 57-69.

DELIBERATO, D.; GONCALVES, M. J.; MACEDO, E. C. (Orgs.). Comunicação alternativa: teoria, prática, tecnologias e pesquisa. São Paulo: Memnon; 2009.

GONÇALVES, M.J.; CAPOVILLA, F.C.; MACEDO, E. C. Comunicação alternativa, tecnologia e fonoaudiologia. In: Foz FB, Piccarone MLCD, Bursztyn CS (Org.). A tecnologia informática na fonoaudiologia. São Paulo: Pelxus; 1998, p. 114-126.

GUEDES, V.; BORSCHIVER, S. Bibliometria: uma ferramenta estatística para a gestão da informação e do conhecimento, em sistemas de informação, de comunicação e de avaliação científica e tecnológica. In: Proceedings CINFORM - Encontro Nacional de Ciência da Informação VI, Salvador, Bahia, 2005.

HAYASHI, M. C. P. I.; HAYASHI, C. R. M.; MARTINEZ, C. S. Estudos sobre jovens e juventudes: diferentes percursos refletidos na produção científica brasileira. Educação, Sociedade \& Culturas. v. 27, p. 131-154, 2008.

JANNUZZI, G. M. A educação do deficiente no Brasil: dos primórdios ao inicio do século XXI. Campinas: Autores Associados. 2004.

LIMA,C. S. Eficácia de um programa de comunicação alternativa aplicado a grupos de escolares com deficiência intelectual [Dissertação] São Carlos: Universidade Federal de São Carlos - Programa de Pós-Graduação em Educação Especial; 2009.

MUGNAINI, R.; CARVALHO, T.; CAMPANATTI-OSTIZ, H. Indicadores de produção científica: uma discussão conceitual. In: POBLACION, D. A.; WITTER, G. P.; SILVA, J. F. M. Comunicação \& produção científica: contexto, indicadores e avaliação. São Paulo: Angellara; 2006. p. 313-340.

NORONHA, D. P.; MARICATO, J. M. Estudos métrios da informação: primeiras aproximações; Rev. Eletr. Bibliotecon. Ci. Inf. 2008; n. esp., $1^{\circ}$ sem.

NUNES, L. R. O. P. Favorecendo o desenvolvimento da comunicação em crianças e jovens com necessidades educacionais especiais. Rio de Janeiro: Dunya. Linguagem e comunicação alternativa: uma introdução. 2003, p. 3-13.

NUNES, L. R.; NUNES,D; ARAÚJO, I.; BERNAT, A. B.; NOGUEIRA, D.; PASSOS, M.; VALÉRIO, T.; MAGALHÃES, A.P; MADEIRA, S.; MACEDO, E.; CAPOVILLA, F. Questões relativas à iconicidade dos símbolos usados em comunicação alternativa para portadores de paralisia cerebral e deficiência mental. Caderno do Fórum Permanente de Educação e Saúde do Instituto Philippe Pinel; 2000. p. 69-77. 
NUNES, L. R. O. P.; AZEVEDO, M. O.; FREIRE, J. G. Comunicação alternativa em sala de aula: relatos de uma professora de alunos com autismo. In: NUNES, L. R. O. P.; PELOSI, M. B.; WALTER, C. C. F. Compartilhando experiências: ampliando a comunicação alternativa. Marilia: Editora da ABPEE; 2011, p. 161-173.

NUNES, L. R. O. P.; DELGADO, S. M. M.; WALTER, C. C. F. O que dizem as famílias e os profissionais sobre a comunicação alternativa. In: NUNES, L. R. O. P.; PELOSI, M. B.; WALTER, C. C. F. Compartilhando experiências: ampliando a comunicação alternativa. Marilia: Editora da ABPEE; 2011, p. 41-55.

PANHAN, H. M. S. A clínica com adultos e a comunicação alternativa: experiências compartilhadas apoiadas na CIF. In: NUNES,L.R.O.P.; PELOSI, M.B.; WALTER, C. C. F. Compartilhando experiências: ampliando a comunicação alternativa. Marília: ABPEE; 20ll, p. $17-27$.

PAULA, K. M. P. A linguagem funcional em crianças com deficiência múltipla através de sistemas de comunicação alternativa e do ensino naturalístico [Dissertação]. Rio de Janeiro: Universidade do Estado de Rio de Janeiro; 1998.

PELOSI, M. B. A Comunicação alternativa e ampliada nas escolas do município do Rio de Janeiro. In: NUNES, L. R. P. (Org.). Favorecendo o desenvolvimento da comunicação em crianças e jovens com necessidades educacionais especiais. Rio de Janeiro: Dunya; 2003, p. 63-75.

PIZA, M. H. M. O uso dos métodos alternativos PECS-Adaptado e PCS para aumentar habilidades comunicativas em paralíticos cerebrais, não verbais [Dissertação]. São Carlos: Universidade Federal de São Carlos - Programa de Pós-Graduação em Educação Especial; 2002.

REILY, L. Comunicação suplementar e alternativa no contexto da inclusão. In: MARTINS, L. A. R.; et al. (Orgs.). Práticas inclusivas no sistema de ensino e em outros contextos. Natal: Ed. da UFRN; 2009, p. 211-221.

SAMESHIMA, F. S.; DELIBERATO, D. Habilidades comunicativas utilizadas por um grupo de alunos não-falantes durante atividade de jogo. In: NUNES, L. R. O. P.; PELOSI, M. B.; GOMES, M. R. (Org.). Um retrato da comunicação alternativa no Brasil: relatos de pesquisas e experiências. Rio de Janeiro: 4 Pontos Estúdio Gráfico e Papéis; 2007, p. 118-122.

SILVA, M. R. Análise bibliométrica da produção científica do Programa de Pós-graduação em Educação Especial da UFSCar. 1998 - 2003 [Dissertação]. São Carlos: Universidade Federal de São Carlos; 2004.

SILVA, M. R.; HAYASHI, M. C. P. I.; HAYASHI, C. R. M. Análise bibliométrica e cientométrica:desafios para especialistas que atuam no campo. In: CID: R. Ci. Inf. e Doc. 2011; 2(1):110-129.

WALTER, C. C. F. Avaliação de um programa de comunicação alternativa e ampliada para mães de adolescentes com autismo [Tese]. São Carlos: Universidade Federal de São Carlos Programa de Pós-Graduação em Educação Especial; 2006.

WALTER. C.; ALMEIDA, M. A. Avaliação de um programa de comunicação alternativa e ampliada para mães de adolescentes com autismo. Rev. Bras. Ed. Esp. 16(3), p. 429-446, 2010. 


\section{Anexo}

Anexo I - Lista dos 24 artigos analisados

ALLEN, J. Linguistic aspects of speech synthesis. Proc. Natl. Acad. Sci. USA.1995;92:99469952.

ALMEIDA, M. A.; PIZA, M. H. M.; LAMÔNICA, D. A. C. Adaptações do sistema de comunicação por troca de figuras no contexto escolar. Pró-fono R. Atual.Cient. 17(2), p. 233-240, 2005.

BIRBAUMER, N.; COHEN, L. G. Brain-computer interfaces: communication and restoration of movement in paralysis. J Physiol. 579(3), p. 621-636, 2007.

CARLSON, R. Models of speech synthesis. Proc. Natl. Acad. Sci. USA.1995;92:9932-9937.

CHARLOP-CHRISTY, M. H.; CARPENTER, M. L. E. L.; LEBLANC, L. A.; KELLET, K. Using the picture exchange communication system (pecs) with children with autism: assessment of pecs acquisition, speech, social-communicative behavior, and problem behavior. JABA. 35(3), p. 213-231, 2002.

DONOGHUE, J. P.; NURMIKKO, A.; BLACK, M.; HOCHBERG, L. R. Assistive technology and robotic control using motor cortex ensemble-based neural interface systems in humans with tetraplegia. J Physiol. 579(3), p. 603-611, 2007.

DURAND, V. M. Functional communication training using assistive devices: recruiting natural communities of reinforcement. JABA. 32(3), p. 247-267, 1999.

EASTON, J. Communication aids. BMJ. 296, p. 193-195, 1988.

FIDALGO, A. P.; GODOI, J. P.; GIOIA, O. S. Análise de um procedimento de comunicação funcional alternativa (pictureexchange communication system). Rev. Bras. de Ter. Comp. Cogn. 10(1), p. 51-66, 2008.

HUSTAD, K .C.; KEPPNER, K.; SCHANZ, A.; BERG, A. Augmentative and Alternative Communication for Preschool Children: Intervention goals and use of technology. Semin Speech Lang. 29(2), p. 83-91, 2008.

HURLBUT, B. I.; IWATA, B. A.; GREEN, J. D. Nonvocal language acquisition in adolescents with severe physical disabillities: blissymbol versus iconic stimulus formats. JABA. 15(2)p. 241-258, 1982.

KUBLER, A.; BIRBAUNER, N. Brain-Computer Interfaces and communication in paralysis: extinction of goal directed thinking in completely paralysed patients? Clin. Neurophysiol. 119(11), p. 2658-2666, 2008.

KURLAND, J.; NAESER, M. A.; BAKER, E. H.; DORON, K.; MARTIN, P. I.;SEEKINS, H. E.; BOGDAN, A.; RENSHAW P. YURGELUN-TODD D. Test-retest reliability of fMRI during nonverbal semantic decisions in moderate-severe nonfluent aphasia patients. Behav Neurol. 15(3-4), p. 87-97, 2004. 
LEE, R. E. The personal computer: a new friend for the handicapped. CMAJ. 135, p. 10731076, 1986.

LIBERMAN, M. Computer speech synthesis: Its status and prospects. Proc. Natl. Acad. Sci. USA. 92, p. 9928-9931, 1995.

LINEBARGER, M.; DENISE, M. C.;CALL, D.; VIRATA, T.; BERNDT, R. S. Widening the temporal window: Processing support in thetreatment of aphasic language production. Brain Lang. 100(1), p. 53-68, 2007.

PAUL, R. Interventions to Improve Communication.Child AdolescPsychiatrClin N Am. 17(4), p. 835, 2008.

PERRY, A. R.; GAWEL, M.; ROSE, F. C. Communication aids in patients with motor neurone disease. BMJ. 1981; 282:1690-1692.

SCHEPIS, M. M.; REID, D. H. Effects of a voice output communication aid on interactions between support personnel and an individual with multiple disabilities. JABA. 28(1), p. 73-77, 1995.

SCHLOSSER, R. W.; BELFIORE, P. J.; NIGAM, R.; BLISCHAK, D.; HETZRONI, O. The effects of speech output technologyin the learning of graphic symbols. JABA. 28(4), p. $537-549,1995$.

SEELBACH, C. A perspective on early commercial applications of voice processing technology for telecommunications and aids for the handicapped. Proc. Natl. Acad. Sci. USA.1995;92:9989-9990.

TETZCHNER, S. V.; BREKKE, K. M.; SJØTHUN, B.; GRINDHEIM, E. Inclusão de crianças em educação pré-escolar regular utilizando comunicação suplementar e alternativa. Rev. Bras. Ed. Esp. 11(2), p. 151-184, 2005.

WACKER, D. P.; WIGGINS, B.; FOWLER, M.; BERG, W. K. Training students with profound or multiple handicaps to make requests via microswitches. JABA. 21(4), p. 331$343,1988$.

WOLPAW, J. R. Brain-computer interfaces as new brain output pathways. J Physiol. 579(3), p. 613-619, 2007.

\section{Correspondência}

Cândice Lima Moreschi - Rua Antônio de Godoy, 3222 Apto. 32 Centro, CEP: 15015-100, São José do Rio Preto, São Paulo, Brasil.

E-mail: candicelima@yahoo.com.br - suzebello@gmail.com-dmch@ufscar.br

Recebido em 14 de maio de 2012

Aprovado em 13 de agosto de 2012

Revista Educação Especial | v. 26 | n. $47 \mid$ p. 665-684 | set./dez. 2013 